\title{
Exponential Distributions in a Mechanical Model for Earthquakes
}

\author{
M. de Sousa Vieirat \\ Departamento de Fisica, Universidade Federal do Rio Grande do Norte, 59072-970 Natal, RN, \\ Brazil \\ Department of Electrical Engineering and Computer Sciences, University of California, Berkeley, \\ CA 94720, USA.
}

\begin{abstract}
We study statistical distributions in a mechanical model for an earthquake fault introduced by Burridge and Knopoff [R. Burridge and L. Knopoff, Bull. Seismol. Soc. Am. 57, 341 (1967)]. Our investigations on the size (moment), time duration and number of blocks involved in an event show that exponential distributions are found in a given range of the paramenter space. This occurs when the two kinds of springs present in the model have the same, or approximately the same, value for the elastic constants. Exponential distributions have also been seen recently in an experimental system to model earthquake-like dynamics [M. A. Rubio and J. Galeano, Phys. Rev. E 50, $1000(1994)]$
\end{abstract}

Typeset using REVTEX 


\section{INTRODUCTION}

Systems that present stick-slip dynamics and scaling invariance have attracted considerable attention recently [1,2]. This was triggered, in part, by a seminal paper of Bak, Tang and Wiesenfeld, which showed that a class of systems presenting avalanches ou earthquakelike dynamics can naturally attain a critical state characterized by power-law distributions [1]. They denoted this phenomenon Self-Organized Criticality.

Recently, two experimental studies in a continuous elastic system have been performed to model the stick-slip dynamics observed in earthquake-like phenomena. In one of them [3], a glass rod is pulled on top of a latex membrane. In this system the slipping events appear as detachment waves and a wide distribution of event sizes was observed. However, robust scaling behavior was not seen.

In the other system [4], the elastic medium consists of a transparent gel sheared between two coaxial circular cylinders. The inner cylinder is rotated at very low angular speed. Thus, this system closely resembles the physics of a spring-block model [5] with periodic boundary conditions. Due to the friction between the gel and the cylinders, stick-slip behavior is seen in the form of detachment waves. Rubio and Galeano were able to identify four regimes in this system, by varying the gel's rigidity, the rotor angular speed and the friction properties of the gel with the inner cylinder. The regimes identified were: (a) uniform slipping of the gel with respect to the rotor; (b) relaxation events, in which a big event starts to quickly propagates through the whole cell in an almost periodic way; (c) nearly periodic regimes of a soliton-like character and (d) a regimes involving events with many size and time scales. In the latter case, they found exponential statistical distributions in amplitude, duration and separation time between events.

With respect to theoretical and numerical studies, one of the systems used to model the dynamics of earthquakes has been the model introduced by Burridge and Knopoff (BK) in 1967, and investigated extensively in recent publications [6,7]. Although most of the studies on the Burridge-Knopoff model have been concentrated on the parameter region where a 
partial power-law (partial here means of limited size) of the distributions of event sizes is found, this regime in fact has not been seen yet in experimental studies of a homogeneous system. The three first regimes (a), (b) and (c) found by Rubio and Galeano have been observed in numerical studies of the BK model. No reports so far exist of exponential distributions in the BK model.

In this paper we show that exponential distributions are also seen in the BK model. This kind of distribution is observed when the two kinds of springs in the model have the same, or approximately the same, value for the elastic constants. They are present in quantities such as the time duration, number of blocks displaced, and total displacement (moment) of the blocks involved in an event.

The paper is organized as follows: in the next section we describe the model; in the third section we study the statistical distributions and the different regimes that appear when the spring constants are varied; the last section is dedicated to the conclusions.

\section{THE BURRIDGE-KNOPOFF MODEL}

The homogeneous version of the Burridge and Knopoff model is shown in Fig. 1 of Ref. [6]. It consists of a one dimensional array of $N$ blocks, each of mass $m$, coupled by springs of constant $k_{c}$ to one another, and by a spring of constant $k_{p}$ to a rigid pulling bar that moves at constant velocity $V$. In equilibrium, when all the springs are unstretched, adjacent blocks are separated by a distance $a$. The blocks rest upon a stationary surface, which provides a frictional force that impedes the motion of the blocks. In the version considered in Ref. [6] the friction is a decreasing function of the velocity, the same for all blocks. The equation of motion for the $j$ th mass when it is moving is

$$
m \ddot{X}_{j}=k_{c}\left(X_{j+1}-2 X_{j}+X_{j-1}\right)-k_{p}\left(X_{j}-V t\right)-F\left(\dot{X}_{j} / V_{f}\right), \quad \dot{X}_{j} \neq 0,
$$

where $X_{j}$ denotes the displacement of the block measured with respect to the position where the sum of the elastic forces on it is zero. The last term in Eq. (11) represents the nonlinear and velocity dependent frictional force, which is given by 


$$
F\left(\dot{X}_{j} / V_{f}\right)=\left\{\begin{array}{cl}
F_{0} /\left(1+\dot{X}_{j} / V_{f}\right), & \text { if } \dot{X}_{j}>0 \\
-\infty, & \text { if } \dot{X}_{j}<0
\end{array}\right.
$$

where $V_{f}$ is the characteristic velocity for the friction. Here, we do not allow backward motions and the friction force gets arbitrarily high to avoid it. This is just for computational convenience, and this does not change the main results presented here.

As shown in Ref. [7], this model has five independent velocity scales. Two appear explicitly in Eq. (1): (i) the pulling velocity $V$ and (ii) the characteristic friction $V_{f}$. Two more can be defined in terms of the spring constants, the mass and $F_{0}$ : (iii) $V_{0}=F_{0} / \sqrt{m k_{p}}$ and (iv) $V_{l}=\left(F_{0} / k_{p}\right) \sqrt{k_{c} / m}$. Here, $V_{0}$ and $V_{l}$ correspond to the maximum velocities of a single block held by a spring of constant $k_{p}$ and $k_{c}$, respectively, when it has displaced by the characteristic distance $F_{0} / k_{p}$ in the absence of friction. The fifth velocity is the sound velocity $V_{s}=a \sqrt{k_{c} / m}$, which depends on the equilibrium spacing of the blocks $a$ and does not appear explicitly in Eq. (11). Depending on the relative sizes of these velocities, we would expect different behavior for the system.

Following Ref. [6], we introduce different dimensionless variables

$$
U_{j}=\frac{k_{p}}{F_{0}} X_{j}, \quad \tau=\left(m / k_{p}\right)^{1 / 2} t,
$$

so that Eq. (11) can be written in dimensionless form

$$
\ddot{U}_{j}=\nu_{l}^{2}\left(U_{j+1}-2 U_{j}+U_{j-1}\right)-U_{j}+\nu \tau-\Phi\left(\dot{U}_{j} / \nu_{f}\right)
$$

where

$$
\Phi\left(\dot{U}_{j} / \nu_{f}\right)=\left\{\begin{array}{cl}
1 /\left(1+\dot{U}_{j} / \nu_{f}\right), & \text { if } \dot{U}_{j}>0 \\
-\infty, & \text { if } \dot{U}_{j}<0
\end{array}\right.
$$

Now, $\nu_{l}=\sqrt{k_{c} / k_{p}}, \nu=V / V_{0}$, and $\nu_{f}=V_{f} / V_{0}$. Dots here represent derivatives with respect to the scaled time $\tau$. The sound velocity becomes $\nu_{s}=a^{\prime} \sqrt{k_{c} / k_{p}}=a^{\prime} \nu_{l}$, where $a^{\prime} \equiv a k_{p} / k_{c}$. Without losing generality we can take the dimensionless length $a^{\prime} \equiv 1$. Thus, the sound velocity $\nu_{s}$ becomes identical to $\nu_{l}$. The five velocities $V, V_{f}, V_{l}, V_{s}$ and $V_{0}$ have been 
transformed respectively into $\nu, \nu_{f}, \nu_{l}, \nu_{s} \equiv \nu_{l}$, and $\nu_{0} \equiv 1$. Consequently, the model has three relevant parameters, $\nu, \nu_{f}$ and $\nu_{l} \equiv \nu_{s}$, which completely determine the behavior of the system. In the case of open boundary condition, which is the one we consider here, we have $U_{0}=U_{1}$ and $U_{N+1}=U_{N}$.

For fixed $\nu_{l}$ and $\nu$ (large $\nu_{l}$ and small $\nu$ ), a transition was reported in Ref. [7] when two velocities cross, namely, when $\nu_{f}=\nu_{0} \equiv 1$. For $\nu_{f}=\infty$ one finds that the motion of the system is continuous. No block ever stops. As $\nu_{f}$ decreases, one sees small regions of stationary blocks. When $\nu_{f}$ becomes less than 1 these stationary (event-free) regions begin to percolate across the entire system. The motion of the fault in this small $\nu_{f}$ region now occurs in abrupt large events.

Here we report another transition, which occurs when the the velocity $\nu_{l}$ is varied for fixed values of $\nu$ and $\nu_{f}$. We find that for $\nu_{l}$ less than a given value the event sizes and event durations obey exponential distributions. Another value is observed for $\nu_{l}$, above which the distributions present partial power-laws, namely, events that have size smaller than a critical value have power-law distributions, and events that are larger than this value obey different statistics. In the intermediate regime for $\nu_{l}$, the distributions are neither powerlaws nor exponentials. As $\nu_{l}$ gets close to zero, the exponential distributions disappear, since in that limit the blocks are disconnected, and only one-block events are observed. Evidence of a different behavior when $\nu_{l}$ is varied was found in Ref. [8]. However, no statistical distributions were calculated in that paper.

In the numerical simulations of this letter we have generally started the system with the blocks at rest and with the sum of the elastic forces in each block equal to zero. Other initial conditions were considered and the results did not change. We have also considered periodic boundary conditions, and the results were the same (except for the few events involving the boundaries of the chain). Before we start to compile statistics we let the system evolve until it reaches a statistical stationary state. Here we fix the pulling velocity and the characteristic velocity to the following values: $\nu=0.01$ and $\nu_{f}=1 / 6$. It is beyond the scope of this paper to do a detailed study on the effects of variations in the pulling 
velocity and in the characteristic speed. Studies on event distributions for varying $\nu_{f}$ and $\nu$ with fixed $\nu_{l}$ can be found in [6]. We expect that the kind of transition we report here will be observed for other values of $\nu_{f}$ and $\nu$, and the value of $\nu_{l}$ where the transition occurs probably varies with the characteristic and pulling velocities.

\section{NUMERICAL RESULTS}

To illustrate the different behaviors of the system when $\nu_{l}$ is varied, we show in Fig. 1 projections of the block velocities $\dot{U}_{j}$ onto the $j-\tau$ plane. A black dot in the figure means that the $j$-block is moving at time $\tau$ and a white one, that it is at rest. We show three different cases with $\nu_{l}=1,3,10$. It is clear that a transition occurs as $\nu_{l}$ is changed. For small $\nu_{l}$, we see clusters of moving blocks, which involve only a small number of masses. As $\nu_{l}$ is increased, one finds clusters involving a small number of blocks, as well as clusters involving a large number of masses. These large ruptures appear $\vee$ shaped and travel with the sound speed $\nu_{l}$.

Now we investigate the distributions of moment and time duration for different values of $\nu_{l}$. The moment of an earthquake is a measure of its size, and in dimensionless units it is defined as $m=\sum_{j} \delta U_{j}$, where $\delta U_{j}$ is the displacement of the $j$-th block and the sum is over the blocks displaced during the event. The distribution $r(m)$ is the number of events with magnitude $m$ divided by the number of blocks $N$ in the chain and by the total time of dynamical evolution. For $\nu_{l} \lesssim 2$ we find that $r(m)$ is governed by an exponential function, namely, $r(m) \sim \exp \left(m / m_{c}\right)$, where $m_{c}$ is a characteristic moment. The result of $r(m)$ with $\nu_{l}=1$ is shown in Fig. 2(a) as a solid line. We have also found that the characteristic moment decreases as $\nu_{l}$ becomes smaller, since when $\nu_{l}=0$ the blocks become disconnected and only events involving one block are observed. For intermediate values of $\nu_{l}\left(2 \lesssim \nu_{l} \lesssim 4\right)$ ones sees a regime for $r(m)$ which is neither power-law nor exponential behavior. This is shown in Fig. 2(b) where the solid line represents $r(m)$ for $\nu_{l}=3$. For large $\nu_{l}\left(\nu_{l} \gtrsim 4\right)$ one sees the appearance of a partial power-law distribution. This was the parameter region 
investigated by Carlson and Langer [6]. Beyond the power-law cutoff one sees a bump in the distributions, which increases in height as $\nu_{l}$ becomes larger. We have found that the events that do not belong to the scaling region become more and more frequent as $\nu_{l}$ is increased. Thus, in the limit of very large $\nu_{l}$ the power-law tends to disappear. In Fig. 2(c) the solid line represents $r(m)$ for $\nu_{l}=10$.

For the distributions of time durations $\delta \tau$ of the events we find qualitatively the same kind of behavior as found for the distributions of moments. For $\nu_{l} \lesssim 2$ we get an exponential function, $r(\delta \tau) \sim \exp \left(\delta \tau / \delta \tau_{c}\right)$. This is shown in Fig. 2(a) for $\nu_{l}=1$ as a dashed line. Again, we have found that the characteristic time duration $\delta \tau_{c}$ decreases as $\nu_{l}$ gets smaller. For $\nu_{l} \gtrsim 4$ one sees partial power-law distributions, as shown in Fig. 2(c) for $\nu_{l}=10$ as a dashed line. For intermediate values of $\nu_{l}\left(2 \lesssim \nu_{l} \lesssim 4\right)$ the distributions are neither exponential nor power-law ones, as we show in Fig. 2(b) for $\nu_{l}=3$ (dashed line).

We have also studied the statistical distributions $r(n)$ of an event involving $n$ blocks. Again, the same kind of behavior described above is seen, namely, exponential distributions for $\nu_{l} \lesssim 2$, partial power-laws for $\nu_{l} \gtrsim 4$, and a regime without any scaling features for intermediate values of $\nu_{l}$.

\section{CONCLUSIONS}

We have found exponential distributions for the sizes and time durations of the events in the Burridge-Knopoff model for earthquakes when the two kinds of springs have the same (or approximately the same) elastic constants. In this situation the velocity $\nu_{l}$ and the sound velocity are equal (or approximately equal) to one. For intermediate values of $\nu_{l}$, the distributions are neither exponential nor power-law. For large $\nu_{l}$, partial power-laws (namely, a scaling region of limited size) are observed. Since in the experimental system studied by Rubio and Galeano [4] the elastic gel is homogeneous, this implies that the exponential distributions they found are consistent with our results for the case in which the springs of the BK model have identical elastic constants. 


\section{REFERENCES}

* Present Address: Dept. of EECS - Cory Hall, University of California, Berkeley, CA 94720. Electronic address: mariav@eecs.berkeley.edu

[1] P. Bak, C. Tang, and K. Wiesenfeld, Phys. Rev. Lett. 59, 381 (1987); Phys. Rev. A 38, 364 (1988).

[2] M. de Sousa Vieira, Phys. Rev. A 46, 6288 (1992).

[3] D. P. Vallete and J. P. Gollub, Phys. Rev. E 47, 820 (1993).

[4] M. A. Rubio and J. Galeano, Phys. Rev. E 50, 1000 (1994).

[5] R. Burridge and L. Knopoff, Bull. Seismol. Soc. Am. 57, 341 (1967).

[6] J. M. Carlson and J. S. Langer, Phys. Rev. Lett. 62, 2632 (1989); Phys. Rev. A 40, 6470 (1989).

[7] G. Vasconcelos, M. de Sousa Vieira and S. R. Nagel, Physica A 191, 69 (1992); M. de Sousa Vieira, G. Vasconcelos and S. R. Nagel, Phys. Rev. E 47, R2221 (1993).

[8] P. Español, Phys. Rev. E 50, 227 (1994).

[9] M. de Sousa Vieira, H. J. Herrmann, Phys. Rev. E 494534 (1994). 


\section{FIGURES}

FIG. 1. Projections of the block velocities $\dot{U}_{j}$ onto the $j-\tau$ plane for a 200 block system with $\nu=0.01, \nu_{f}=1 / 6$, and (a) $\nu_{l}=1$, (b) $\nu_{l}=3$, and (c) $\nu_{l}=10$.

FIG. 2. Statistical distribution $r(m)$ of the moment of the events (solid lines) and the distribution $r(\delta \tau)$ of the time durations of the events (dashed lines) for (a) $\nu_{l}=1$, (b) $\nu_{l}=3$ and (c) $\nu_{l}=10$. In all cases, $N=300, \nu=0.01$ and $\nu_{f}=1 / 6$. 
FIG. 1 (a)

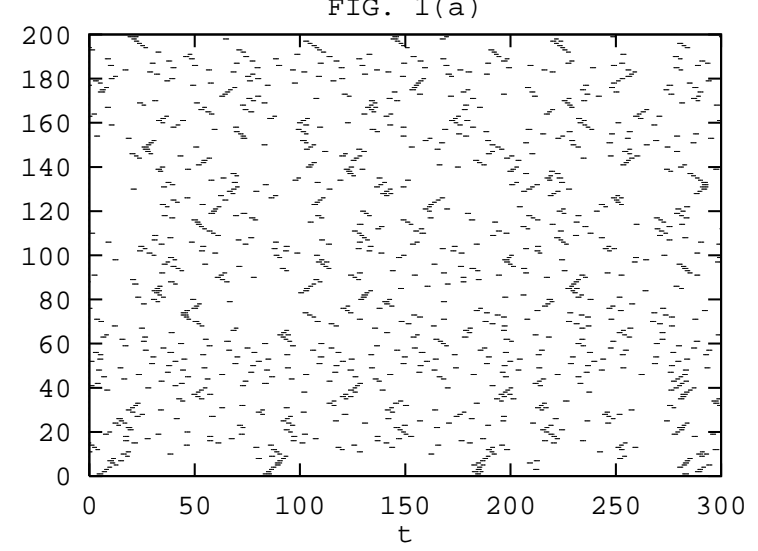

$\cdot \sqcap$

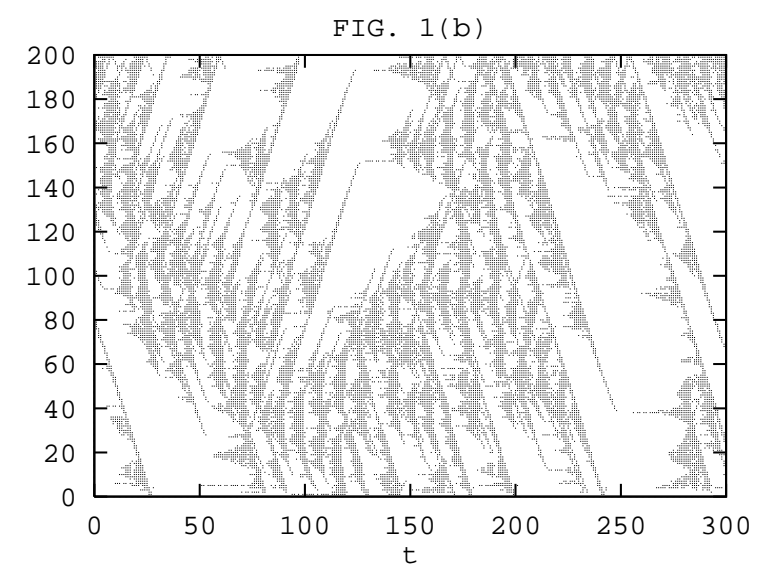

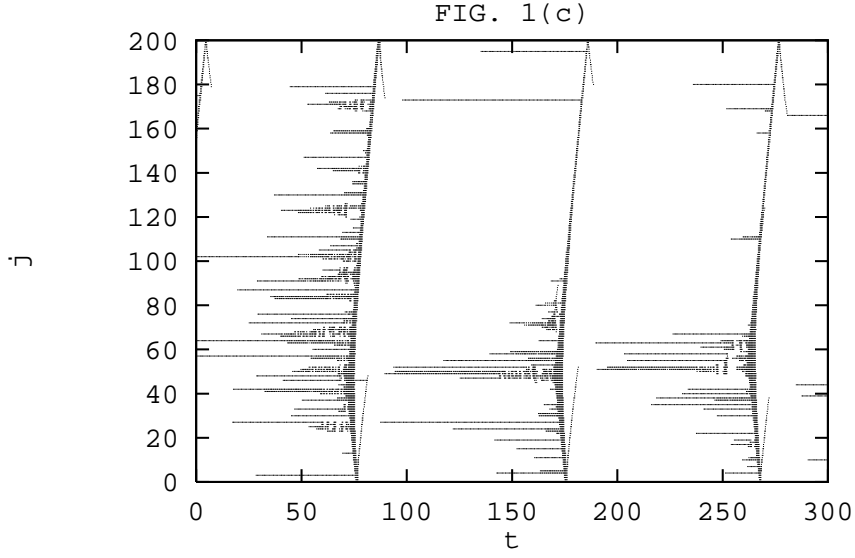

